\title{
Analysis of the Effect of Duloxetine in the Treatment of Early Pain After Total Knee Arthroplasty
}

Tao Wang (D 13821177994@163.com)

Department of bone and joint, Tianjin Beichen Hospital, Tianjin, China

Sen $\mathrm{He}$

Department of bone and joint, Tianjin Beichen Hospital, Tianjin, China

Research article

Keywords: Duloxetine, Total knee replacement, Pain

Posted Date: October 6th, 2020

DOI: https://doi.org/10.21203/rs.3.rs-77484/v1

License: (c) (1) This work is licensed under a Creative Commons Attribution 4.0 International License.

Read Full License 


\section{Abstract}

OBJECTIVE: Total knee arthroplasty is one of the most effective treatments for advanced degeneration of the knee. Knee replacement surgery has achieved excellent clinical results, not only solving the patients' pain, but also solving the social problems. The objective of this study was to examine the effect of duloxetine, a selective serotonin norepinephrine reuptake inhibitor, on the treatment of early pain in patients after TKA.

METHODS: The study is a randomized controlled trial. Sixty patients who underwent TKA from January 2020 to June 2020 were selected for inclusion in the study, and equally assigned to a celecoxib group and a duloxetine group. There were 22 males and 38 females; the average age was $60.3 \pm 5.1$ (56-74) years. The celecoxib group was given celecoxib $200 \mathrm{mg} / \mathrm{bid}$. The duloxetine group received duloxetine $30 \mathrm{mg} / \mathrm{bid}$. Analgesic medication was applied from the first postoperative day in the two groups for 4 weeks. The effectiveness of analgesia was assessed by the postoperative VAS walking pain score, HAMD-17 (Hamilton Depression Scale) score and the WOMAC pain score (Western Ontario and McMaster Universities Osteoarthritis Index, WOMAC). The proportion of adverse events in the two groups was also compared to further evaluate the analgesic effect and safety.

RESULTS: There was no significant difference in VAS score between groups on postoperative day three but duloxetine was superior to the celecoxib group at postoperative weeks one, two and four $(p<0.05)$. The HAMD-17 scores in both groups decreased at week four compared to preoperative values $(p<0.05)$, but the duloxetine group's HAMD-17 score was superior to the celecoxib group four weeks postoperatively $(P<0.05)$. The WOMAC pain scores of the duloxetine group were superior to the celecoxib group $(P<$ 0.05 ) at postoperative weeks one, two and four. There was no significant difference in the incidence of adverse events.

CONCLUSION: Duloxetine has a good effect on postoperative analgesia after TKA and can relieve patients' depression and anxiety, and promote rapid recovery. Thus, we can infer that duloxetine can be used to treat early postoperative pain after knee arthroplasty.

\section{Introduction}

Total knee replacement is a successful surgical procedure for the treatment of advanced knee arthritis, improving the function of the patient's joint and meeting the needs of a large number of patients to live their daily lives ${ }^{[1]}$, which in turn improves their quality of life. With the rise of rapid rehabilitation joint surgery after knee replacement, short-term, high quality integrated management of perioperative pain, blood management, infection prevention and dietary management can achieve an optimal effect.

Many studies have shown a $90 \%$ survival rate after 10 years for knee replacements, but there are still some patients who are dissatisfied with their postoperative results, primarily pain. Postoperative pain after joint replacement remains a challenge ${ }^{[2]}$. It can affect recovery and rehabilitation, as well as prolong the length of hospitalization and increase the financial burden on patients. Because of this, multimodal 
analgesia was developed. Among these analgesics, oral analgesics play an important role. In the past, commonly used analgesics were mainly opioids, but these drugs have serious side effects such as respiratory depression and nausea. Non-steroidal anti-inflammatory drugs (NSAIDs) are widely used to reduce complications and achieve good pain relief. These drugs can inhibit the activity of cyclooxygenase and the production of prostaglandins from arachidonic acid ${ }^{[3]}$, and control pain but prolonged use can also bring cardiovascular complications. At the same time, in recent years, with the improvement of people's living standards, psychological conditions also have received more attention. A survey found that the incidence of depression among diabetic patients is two times that of non-diabetic people, $30 \%$ of coronary heart disease patients suffer from depression, and there is a positive correlation between the severity of knee arthritis and the prevalence of depression ${ }^{[4]}$. Chronic pain also can lead to depressive symptoms and can affect the patient's recovery after surgery.

Blackburn-Munro reported that about $50 \%$ of pain patients had mood disorders, and Thomson et al. found that $22 \%$ of Canadian patients with fibromyalgia syndrome suffered from major depression. Several studies now suggest that depression and chronic pain share a common pathogenesis and there may be a genetic link between the two. The common pathogenesis may be related to several brain regions, including the hippocampus, insular cortex, anterior cingulate gyrus, cerebellum, prefrontal cortex, and hypothalamus. In addition, depression has a strong influence on the subjective perception of pain , as patients with depression have a lower pain threshold than ordinary patients.

The majority of patients with end-stage knee osteoarthritis can be treated with TKA to relieve pain and restore joint function, but not all patients who undergo surgery have a good prognosis. It has been reported that pain in patients with chronic knee arthritis is secondary and continues to cause pain even after TKA. This pain has peripheral and central nociceptive sensitization ${ }^{[5]}$. Peripheral sensitization decreases the threshold of afferent fibers with an upward or leftward shift in the stimulus-response curve and an increase in slope. Central sensitization is defined as pain signaling to the spinal cord and higher centers above the spinal cord. The amplification of the response is a plastic change in the central nervous system in the formation of pain perception. Current studies also suggest that depression and secondary pain share a common pathogenic mechanism: a possible link to the hippocampus, insular cortex, anterior cingulate gyrus, and the insula. Duloxetine is a selective form of serotonin and norepinephrine reuptake inhibitor, in which 5-HT and NE imbalances are associated with central sensitization-associated chronic pain ${ }^{[6]}$. In depressed patients, central serotonin and norepinephrine function are deficient ${ }^{[7]}$, which may impair the function of pain inhibitory pathways. NSAIDs are commonly used analgesics for the treatment of post knee replacement. The most commonly used in our department are selective COX-2 inhibitors (celecoxib) ${ }^{[8]}$. NSAIDs have little effect on the gastrointestinal tract or on blood clotting but can have negative side effects and do not have an effect on depression ${ }^{[9]}$. Antidepressants inhibit the release of excitatory neurotransmitters, blunt nociceptive pathways, and enhance the effects of the downstream inhibitory system; in patients with psychological disorders, they may improve pain by relieving the patient's psychological disorder. The therapeutic dose of antidepressants for chronic pain is much lower than their therapeutic dose for psychiatric disorders. There is also research evidence that the use of 
antidepressants alone in patients without depressive symptoms can also improve pain symptoms, as well as improve patients' fatigue, sleep disturbances, and quality of life. Currently, duloxetine has been used to treat muscular as well as skeletal pain with good results, but there are no clear studies in the treatment of pain after knee replacement. The present study compares duloxetine with celecoxib in the treatment of postoperative pain after TKA and in the treatment of patient depression. The effect of duloxetine on the outcome of rehabilitation after knee arthroplasty also was evaluated.

\section{Methods}

We selected 60 patients who had undergone unilateral total knee replacement from January 2020 to June 2020 at the bone and joint department of the Tianjin Beichen Hospital. There were 22 males and 38 females; the average age was $60.3 \pm 5.1$ (56-74) years. All patients were divided randomly into two equal ( $n=30 /$ group) duloxetine and celecoxib groups. All patients in this study were informed of the procedures and goals of the study and all signed the informed consent for this study. The study was approved by the Medicine Ethics Committee of the Tianjin Beichen Hospital, and was conducted in accordance with the Declaration of Helsinki.

Inclusion criteria included patients $₫$ with unilateral osteoarthritis who were eligible for initial total knee replacement surgery; 『who had no previous mental illness. or \history of relevant drug use; $₫$ and who could comply with the completion of the experiment.

Patients were excluded if they $₫$ had infectious or rheumatoid arthritis; $₫$ were resistant to COX-2 selective inhibitors or opioids; 『had drug allergies; $\$ presented with severe heart or lung disease that prevented surgery; \had a history of alcohol or drug abuse; $₫$ presented with liver or kidney problems; or \had neurological or psychological disorders.

\section{Surgical Technique}

All TKAs were performed by the same senior surgeon using the P.F.C.Sigma (Depuy, Johnson $\otimes$ Johnson). Under general anesthesia and femoral nerve block, a tourniquet was applied to the proximal part of the limb before surgery. Intraoperatively, an anterior median knee incision was used with a parietal medial approach. The proximal tibial osteotomy was performed using extramedullary positioning with the osteotomy surface inclined $7^{\circ}$ posteriorly. The distal femur was osteotomized using intramedullary positioning, with the osteotomy surface rotated $6^{\circ}$ from the anatomic axis of the femur. The anterior and posterior femoral condylar osteotomy was performed using the anterior reference method with $3^{\circ}$ external rotation. The intercondylar femur and tibial plateau were treated, and a prosthesis specimen was fitted. After confirmation of satisfactory flexion/extension clearance and soft tissue balance, the prosthesis was fitted and cemented. After suturing the joint capsule, $1 \mathrm{~g}$ of tranexamic acid was injected into the joint. None of the patients had patellar replacement. No drains were placed in the wounds. A "cocktail" consisting of ropivacaine, epinephrine and dexamethasone was injected into the joint capsule of all patients. Rivaroxaban (10 mg/qd) was used for all patients for four weeks postoperatively to prevent deep venous thromboembolism. 


\section{Outcome measures}

From postoperative day one, patients were encouraged to walk with a walker and to perform muscle strength training as well as ankle pump exercises in bed. VAS scores for pain on walking were recorded on postoperative day three and postoperatively at weeks one, two and four. Hamilton depression scores were recorded before surgery and at postoperative week four. WOMAC pain scores were recorded postoperatively at weeks one and four. The VAS is a standard scale for pain measurement using a $10-\mathrm{cm}$ instrument. Each subject rated knee pain on a scale of 0 to 10, where zero indicates the absence of pain and 10 indicates the worst pain imaginable. The Hamilton Depression Scale is the most commonly used clinical scale to assess the severity of depression and the effectiveness of treatment. A total score $<7$ indicates a normal mental state; a total score of 7-17 indicates a probable depression; a total score of 1724 indicates a definite depression; and a total score of $>24$ indicates a severe depression. The WOMAC score is used to assess knee function. We used the WOMAC pain rating scale, which consists of five questions, each of which is rated on a VAS scale from 0 to 100, with higher scores indicating more severe pain.

\section{Statistical Analysis}

All statistical analyses are based on SPSS 26.0 (SPSS version 26.0 for Windows; IBM). The normality of continuous variables was tested using the Kolmogorov-Smirnov test. If the variables were normally distributed, Student's $t$ tests were used to compare groups. If the variables were not normally distributed, Wilcoxon signed-ranks tests were used. The chi-square test was used to determine differences in categorical variables. P-values $\mathbb{0} 0.05$ were considered significant.

\section{Results}

There were no significant differences in age, sex, or BMI between treatment groups (Table 1). The VAS score on postoperative day three was not significantly different, but there were significant differences between the two groups at one, two and four weeks postoperatively $(p<0.05)$ (Table 2$)$. HAMD-17 scores in both groups decreased four weeks postoperatively compared to preoperative values $(p<0.05)$, but the HAMD-17 score of the duloxetine group was significantly better than the celecoxib group $(p<0.05)$ (Table 3). WOMAC pain scores were significantly lower in the duloxetine group than in the celecoxib group at all postoperative time points $(p<0.05)$. There were no significant differences in the incidence of adverse events between the two groups.

Table 1. Relevant patient variables 


\begin{tabular}{|llll|}
\hline & Duloxetine group & Celecoxib group & P Value \\
\hline Age $(\mathrm{yr})$ & $64.8 \pm 4.2$ & $65.4 \pm 3.8$ & 0.585 \\
\hline Sex & Male12 & Male 11 & 0.791 \\
\hline & Female 18 & Female 19 & \\
\cline { 1 - 2 }$(\mathrm{kg} / \mathrm{m} 2)$ & $28.5 \pm 1.5$ & $28.6 \pm 1.8$ & 0.82 \\
\hline
\end{tabular}

Table 2. VAS scores for walking (mean $\pm S D$ )

\begin{tabular}{|llll|}
\hline VAS score & Duloxetine group & Celecoxib group & $P$ Values \\
\hline 3 days & $6.1 \pm 0.7$ & $6.1 \pm 0.8$ & 0.735 \\
\hline 1 week & $4.2 \pm 0.9$ & $5.0 \pm 0.6$ & $\bowtie 0.05$ \\
\hline 2 weeks & $3.9 \pm 0.7$ & $4.7 \pm 0.6$ & $\bowtie 0.05$ \\
\hline 4 weeks & $3.4 \pm 0.8$ & $4.1 \pm 0.7$ & $\bigotimes 0.05$ \\
\hline
\end{tabular}

Table 3. Hamildon -17 score (mean $\pm S D$ )

\begin{tabular}{|c|c|c|c|}
\hline HAMD-17 score & Preoperative & postoperative week 4 & P Values \\
\hline Duloxetine group & $12.6 \pm 2.3$ & $7.5 \pm 2.0$ & $\triangle 0.05$ \\
\hline Celecoxib group & $13.6 \pm 2.2$ & $11.4 \pm 2.3$ & $\varangle 0.05$ \\
\hline P Value & 0.08 & $\varangle 0.05$ & \\
\hline
\end{tabular}

Table 4. WOMAC pain scores (mean $\pm S D$ )

\begin{tabular}{|llll|}
\hline WOMAC pain score & Duloxetine group & Celecoxib group & P Values \\
\hline 1 week & $21.3 \pm 2.4$ & $24.4 \pm 3.4$ & $\varangle 0.05$ \\
\hline 2 weeks & $14.7 \pm 2.2$ & $18.5 \pm 3.0$ & $\bigotimes 0.05$ \\
\hline 4 weeks & $8.4 \pm 2.3$ & $11.2 \pm 2.2$ & $\bigotimes 0.05$ \\
\hline
\end{tabular}

Table 5. Adverse events 


\begin{tabular}{|c|c|c|c|}
\hline Adverse events & Duloxetine group & Celecoxib group & P Value \\
\hline dizziness & $3 \otimes 10 \% \bigotimes$ & $4 \rrbracket 13.3 \% \rrbracket$ & 0.688 \\
\hline nausea & $2 \varangle 6.7 \% \rrbracket$ & $3 \rrbracket 10 \% \rrbracket$ & 0.64 \\
\hline drowsiness & $4 \otimes 13.3 \% \rrbracket$ & $3 \rrbracket 10 \% \rrbracket$ & 0.688 \\
\hline
\end{tabular}

\section{Discussion}

Postoperative pain is a challenging complication of TKA, which can result in decreased patient satisfaction and affect rehabilitation ${ }^{[10]}$. The effect can even cause psychological problems such as depression. Duloxetine, a medicine used to treat depression, is increasingly used to treat pain, including lower back pain, muscular pain, fibromyalgia, and pain from osteoarthritis ${ }^{[11,12,13,14]}$. Antidepressants, unlike opioids and NSAIDs, act on 5-HT and NE receptors, which then affect central pain pathways ${ }^{[15]}$. Not only does an anti-depressant upregulate a patient's pain threshold and emotional experience, but it also produces analgesic effects by affecting conductors such as 5-HT and NE and activating opioid receptors ${ }^{[16]}$. Increasingly, research is finding that it also has a peripheral analgesic mechanism ${ }^{[17]}$, which plays a unique role in the treatment of secondary pain ${ }^{[18]}$.

In the present study, duloxetine did not provide significant analgesia in the immediate postoperative period, which may be related to our application of other intra-joint drugs and the use of duloxetine in anesthesia. Femoral nerve block is associated with the ability of duloxetine to provide effective analgesia postoperatively. However, the WOMAC scores one, two and four weeks postoperatively were lower in the duloxetine group than in the celecoxib group, which indicates that postoperative knee function in the duloxetine group was better than in the celecoxib group. Therefore, we can include duloxetine in the current multimodal pain management protocols to treat postoperative pain after TKA.

The prevalence of depression fluctuates between $15-20 \%$ and is characterized by a persistent depressed mood, decreased interest and loss of pleasure ${ }^{[19]}$. The prevalence of depression is about $3 \%$ worldwide, more so in developed countries, and there is still a lack of awareness of depression intervention in society. By the middle of the twenty-first century, it is projected that the disease could become the second leading cause of death and disability in humans ${ }^{[20]}$. Antidepressant measures include psychological interventions and medication, which to date have remained the mainstay of acute or long-term maintenance of depression ${ }^{[21]}$. A retrospective cross-sectional study showed that the symptoms of $67.8 \%$ of 647 patients with osteoarthritis associated with depression who received medication improved [22]. Antidepressants are classified as tricyclics (TCAs), monoamine oxidase inhibitors (MAOIs), 5-HT reuptake Inhibitors (SSRIs), 5-HT and NE reuptake inhibitors (SNRIs), and NE and specific 5-HT antagonists (NE-SSAs). Obviously, not all antidepressants provide adequate pain relief. The TCAs, SSRIs, 
and SNRIs are used clinically to treat secondary pain. Some patients in the current study showed signs of mild depression due to pain and anxiety about the results of surgery during the recovery process ${ }^{[23]}$. Duloxetine is a second generation antidepressant with significantly less drug toxicity than the first generation. Changes in the concentration of certain inflammatory factors in synovial fluid in patients with osteoarthritis of the knee have been reported to be consistent with changes in depression ${ }^{[24]}$. This is further evidence that duloxetine has an anti-inflammatory and analgesic effect on knee arthritis, which will have a positive effect on pain after TKA ${ }^{[25]}$.

A preoperative pain sensitization scale assessment of surgical patients has been proposed in the literature, and the use of duloxetine for analgesia in these patients has achieved significant results [26]. Patients can be grouped so that more targeted medication can be administered. In patients with high central sensitization, the use of NSAIDs is less effective, whereas the use of duloxetine is significantly more effective. However, the distinction between central sensitization relies on scales, with no obvious laboratory indicators to make an objective distinction.

The safety of duloxetine has been well documented in clinical trials in more than 32,000 patients across a range of indications. More than 530,000 patients have been treated with duloxetine worldwide, demonstrating that duloxetine is safe, well tolerated, and effective. A retrospective analysis of different ethnic groups, including Caucasians, Hispanics, Asians, and Blacks showed that duloxetine has a good safety profile in all ethnic groups ${ }^{[27,28]}$. Antidepressants differ from opioids and NSAIDs by acting on 5$\mathrm{HT}$ and NE receptors. NE and 5-HTergic neurotransmitter systems play an important role in the generation, modulation, and maintenance of chronic pain, thereby affecting central pain pathways. They not only upregulate the pain threshold and emotional experience of patients, but also produce analgesic effects through their effects on conductors such as 5-HT and NE and activation of opioid receptors. Peripheral analgesic mechanisms have a unique role to play in the treatment of pain.

Our study found no statistically significant difference in the rate of adverse events between the two groups. Duloxetine is a centrally acting compound, similar to opioids, but with a significantly different mechanism of action and different adverse effects. Our findings suggest that duloxetine may be a useful alternative to central analgesics because it does not increase drug-related adverse effects after TKA.

This study has several limitations. First, it only compares the treatment effect on short-term postoperative pain, but does not compare the analgesic effect 12 weeks or even longer after surgery. Finally, the disease we treated was osteoarthritis, which could be studied in the future studies in patients with rheumatoid arthritis to further compare the analgesic effects of duloxetine.

\section{Conclusion}

In this study, the data suggest that duloxetine is effective in reducing knee pain associated with knee replacement surgery and is well tolerated by patients. Adding duloxetine to post-knee replacement treatment options has the potential to benefit a growing number of patients, many of whom experience 
intolerable adverse events or inadequate analgesia with other classes of drugs. The positive results of this study further support the use of duloxetine as a centrally acting analgesic in the treatment of pain after total knee replacement.

\section{Declarations}

\section{Ethics approval and consent to participate}

Not applicable.

\section{Consent for publication}

Not applicable.

\section{Availability of data and materials}

\section{All data generated or analysed during this study are included in this published article \\ Competing interests}

The authors declare that they have no competing interests.

\section{Funding}

No external funding was received in preparation of this manuscript.

\section{Authors' contributions}

Tao Wang and Sen He participated in the design of the study and research protocol. Tao Wang is responsible for data acquisition, and will conduct statistical analysis, which was involved in the writing, editing and approval of the final manuscript.

\section{Acknowledgements}

Not applicable.

\section{References}

[1] Canovas F, Dagneaux L. Quality of life after total knee arthroplasty. Orthop Traumatol Surg Res. 2018,104(1S):41-46.

[2] Scott CE, Howie CR, MacDonald D, Biant LC. Predicting dissatisfaction following total knee replacement: a prospective study of 1217 patients. J Bone Joint Surg Br. 2010,92(9):1253-1258. 
[3] Carlesso LC, Niu J, Segal NA, et al. The Effect of Widespread Pain on Knee Pain Worsening, Incident Knee Osteoarthritis (OA), and Incident Knee Pain: The Multicenter OA (MOST) Study. J Rheumatol. 2017,44(4):493-498.

[4]Mesci E, Icagasioglu A, Mesci N, Turgut ST. Relation of physical activity level with quality of life, sleep and depression in patients with knee osteoarthritis. North Clin Istanb. 2015,2(3):215-221.

[5] Chappell AS, Ossanna MJ, Liu-Seifert $\mathrm{H}$, et al. Duloxetine, a centrally acting analgesic, in the treatment of patients with osteoarthritis knee pain: a 13-week, randomized, placebo-controlled trial. Pain. 2009,146(3):253-260.

[6] Arnold LM, Lu Y, Crofford LJ, et al. A double-blind, multicenter trial comparing duloxetine with placebo in the treatment of fibromyalgia patients with or without major depressive disorder. Arthritis Rheum. 2004,50(9):2974-2984.

[6] Mesci E, Icagasioglu A, Mesci N, Turgut ST. Relation of physical activity level with quality of life, sleep and depression in patients with knee osteoarthritis. North Clin Istanb. 2015,2(3):215-221.

[7] Kim KW, Han JW, Cho HJ, et al. Association between comorbid depression and osteoarthritis symptom severity in patients with knee osteoarthritis. J Bone Joint Surg Am. 2011,93(6):556-563.

[8] Ohtori S, Orita S, Yamashita M, et al. Existence of a neuropathic pain component in patients with osteoarthritis of the knee. Yonsei Med J. 2012,53(4):801-805.

[9] Lundblad H, Kreicbergs A, Jansson KA. Prediction of persistent pain after total knee replacement for osteoarthritis. J Bone Joint Surg Br. 2008,90(2):166-171.

[10] Bourne RB, Chesworth BM, Davis AM, Mahomed NN, Charron KD. Patient satisfaction after total knee arthroplasty: who is satisfied and who is not? Clin Orthop Relat Res. 2010,468(1):57-63.

[11] Moore RA, Cai N, Skljarevski V, Tölle TR. Duloxetine use in chronic painful conditions--individual patient data responder analysis. Eur J Pain. 2014,18(1):67-75.

[12] Bidari A, Moazen-Zadeh E, Ghavidel-Parsa B, Rahmani S, Hosseini S, Hassankhani A. Comparing duloxetine and pregabalin for treatment of pain and depression in women with fibromyalgia: an openlabel randomized clinical trial. Daru. 2019,27(1):149-158.

[13] Schukro RP, Oehmke MJ, Geroldinger A, Heinze G, Kress HG, Pramhas S. Efficacy of Duloxetine in Chronic Low Back Pain with a Neuropathic Component: A Randomized, Double-blind, Placebo-controlled Crossover Trial. Anesthesiology. 2016,124(1):150-158.

[14] Chappell AS, Desaiah D, Liu-Seifert H, et al. A double-blind, randomized, placebo-controlled study of the efficacy and safety of duloxetine for the treatment of chronic pain due to osteoarthritis of the knee. Pain Pract. 2011,11(1):33-41. 
[15] Beyer JL. The Use of Antidepressants in Bipolar Depression. Handb Exp Pharmacol. 2019,250:415442.

[16] Goldstein DJ, Lu Y, Detke MJ, Hudson J, lyengar S, Demitrack MA. Effects of duloxetine on painful physical symptoms associated with depression. Psychosomatics. 2004,45(1):17-28.

[17] lyengar S, Webster AA, Hemrick-Luecke SK, Xu JY, Simmons RM. Efficacy of duloxetine, a potent and balanced serotonin-norepinephrine reuptake inhibitor in persistent pain models in rats. $J$ Pharmacol Exp Ther. 2004,311(2):576-584.

[18] Pritchett YL, McCarberg BH, Watkin JG, Robinson MJ. Duloxetine for the management of diabetic peripheral neuropathic pain: response profile. Pain Med. 2007,8(5):397-409.

[19] Jesus C, Jesus I, Agius M. Treatment of Depression in patients with Osteoarthritis: the importance of an early diagnosis and the role of Duloxetine. Psychiatr Danub. 2016,28(Suppl-1):149-153.

[20] Stanners MN, Barton CA, Shakib S, Winefield HR. Depression diagnosis and treatment amongst multimorbid patients: a thematic analysis. BMC Fam Pract. 2014,15:124.

[21] Sultana J, Spina E, Trifirò G. Antidepressant use in the elderly: the role of pharmacodynamics and pharmacokinetics in drug safety. Expert Opin Drug Metab Toxicol. 2015,11(6):883-892.

[22] Scopaz KA, Piva SR, Wisniewski S, Fitzgerald GK. Relationships of fear, anxiety, and depression with physical function in patients with knee osteoarthritis. Arch Phys Med Rehabil. 2009;,90(11):1866-1873.

[23] Agarwal P, Pan X, Sambamoorthi U. Depression treatment patterns among individuals with osteoarthritis: a cross sectional study. BMC Psychiatry. 2013,13: 121.

[24]Reikvam AG, Hustad S, Reikvam H, Apelseth TO, Nepstad I, Hervig TA. The effects of selective serotonin reuptake inhibitors on platelet function in whole blood and platelet concentrates. Platelets. 2012,23(4):299-308.

[25] Shi N, Durden E, Torres A, Cao Z, Happich M. Predictors of Treatment with Duloxetine or Venlafaxine XR among Adult Patients Treated for Depression in Primary Care Practices in the United Kingdom. Depress Res Treat. 2012,2012:815363.

[27] Stewart DE, Wohlreich MM, Mallinckrodt CH, Watkin JG, Kornstein SG. Duloxetine in the treatment of major depressive disorder: comparisons of safety and tolerability in male and female patients. $J$ Affect Disord. 2006,94(1-3):183-189.

[28] Wang G, Bi L, Li X, et al. Efficacy and safety of duloxetine in Chinese patients with chronic pain due to osteoarthritis: a randomized, double-blind, placebo-controlled study. Osteoarthritis Cartilage. 2017,25(6):832-838. 